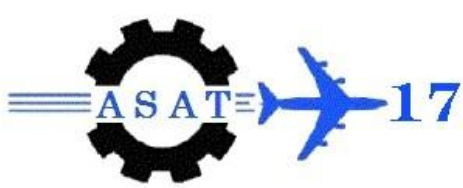

\title{
Optimal Design of Orifice Flow Meter Using Computational Fluid Dynamics (CFD)
}

\author{
Haitham Osman*, Khairy Elsayed ${ }^{\dagger}$, Mohamed El Telbany
}

\begin{abstract}
Orifice flow meter constitutes one of the most common differential pressure flow meters. However, high energy loss is occurred with the measurement process. The present research considers $3 D$ steady simulation using Reynolds Averaged Navier-Stokes equations (RANS). New design of the orifice plate $s$ carried out using the adjoint method. Optimal shape of orifice plate shows $55 \%$ reduction of the pressure loss coefficient at a Reynolds number $R_{e}=1.84 \times 10^{4}$. The discharge coefficient $C_{d}$ is increased by $39 \%$. Also, the effect of downstream ring is investigated. Optimize the shape of the ring has a slight effect on reducing energy consumed. However, applying adjoint method on the orifice plate with downstream ring achieves considerable effect in the pressure loss. Reduction by $34 \%$ in pressure loss coefficient is obtained by the optimal orifice with downstream ring. Shape optimization by the adjoint method has a significant contribution in decreasing the consumed energy through the orifice meter.
\end{abstract}

Keywords: orifice flow meter; pressure loss; optimization; adjoint method.

\section{Introduction}

Many industrial applications require accurate measurement of fluid flow. Several devices have been developed that measure the volumetric fluid flow. Among the devices used for the measurement of discharge are orifice meter, venture meter and nozzle-meter. These meters use differential pressure for the estimation of flow rate. The most common device used for the flow measurement is orifice meter due to its compact size especially for larger flow rates, absence of any moving part and low cost. Some orifice meters are sharp-edged, while others are beveled or rounded. Orifice plate constricts the flow to pass through small cross section. The orifice flow meter employs pressure tappings, both the upstream and the downstream of the orifice plate, for the measurement of pressure drop across the orifice. Streamlines pass through orifice continue to converge beyond the upstream section until they reach minimum area called "vena contracta", Fig. 1. Losses occur in orifice flow meter is considered due to: (1) Formation of vena contracta, due to unrestrained flow contraction. (2) Uncontrolled recovery of pressure. However, the more loss occurs in orifice, the more energy is required in pumping process.

* Demonstrator, Mechanical Power Engineering Department, Faculty of Engineering, Helwan University, Cairo, Egypt. Haithamosman2015@yahoo.com

$\dagger$ Vrije Universiteit Brussel, Department of Mechanical Engineering, Research Group Fluid Mechanics and Thermodynamics, Pleinlaan 2,1050 Brussels, Belgium. khairy.elsayed@vub.ac.be ; Assistant professor, Mechanical Power Engineering Department, Faculty of Engineering, Helwan University, Cairo, Egypt. kelsayed75 @ gmail.com

$\$$ Professor, Mechanical Power Engineering Department, Faculty of Engineering, Helwan University, Cairo, Egypt. profeltelbany@yahoo.com 


\section{Literature review}

The flow through orifice plate gains many interest in literature especially using computational fluid dynamics. Some studies focused on the selection of the turbulence model. Erdal and Andersson [1] investigated numerical aspects of flow through orifice. Investigations were carried out on two-dimensional orifice at $\beta=0.7$ where $\beta$ is diameter ratio $d / D$ (Fig. 1) using different discretization schemes and turbulence models. Due to existence recirculation zone, it is recommended to use low-Reynolds number model. Tunay [2] examined the effects of different numerical methods on the solution of the orifice flow. Orifice flow was investigated by using finite volume method and two finite difference schemes. Imada et al. [3] conducted numerical study utilizing different turbulence models, to determine discharge coefficient $\left(C_{d}\right)$ on an orifice plate. These values were compared with estimated values from ISO (2003). Good agreement was achieved when using SST $k-\omega$ model. Kumar et al. [4] studied 2D, axisymmetric multiple stage orifice plate at various spacing. Eiamsa et al. [5] showed the effects of turbulence model on orifice plate of diameter ratios ( $\beta=0.5,0.6$ and 0.8$)$ on flow field. Several authors (e.g. Siba et al. [6] and Shah et al. [7]) have used CFD to analyze the flow over orifice meter.

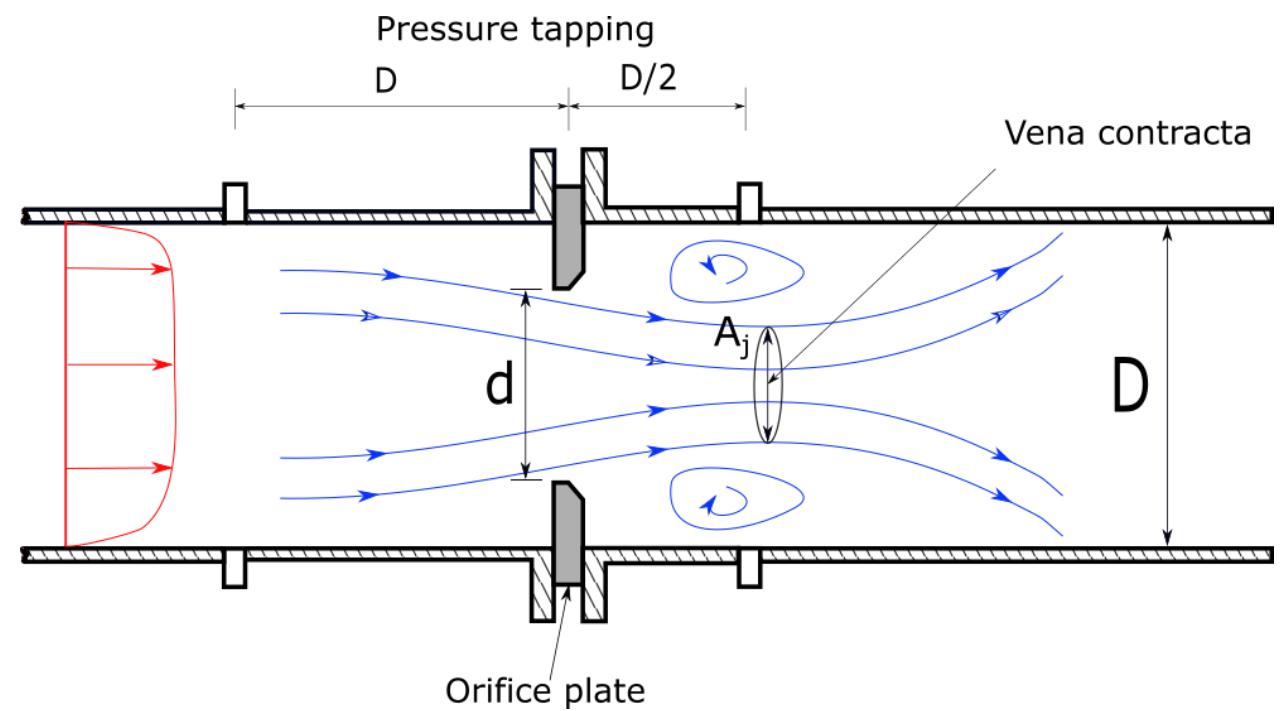

Fig. 1. Schematic of orifice flow

The literature has other studies which focus on minimizing the pressure loss across the orifice meter. An experimental investigation was conducted on fractal shaped orifice. Abou El-Azm et $a l$. [8] show that, the pressure drop after the fractal orifice is less than the pressure drop measured across the circular orifice with the same area. Experimental research by Huang et al. [9] was conducted on perforated orifice as a measuring flow rate. The discharge coefficient of a perforated orifice is $22.5-25.6 \%$ larger than single-hole orifice. Multi-hole orifice plate was explored numerically by Singh et al. [10]. It was observed that the pressure loss is lower than that for a single-hole orifice. Shaaban [11] optimized the geometry of the hole numerically. The results showed that the new hole geometry reduces the pressure loss by $51.7 \%$ at Reynolds number, $R_{e}=3.5 \times 10^{4}$. Also, he stated that using a ring downstream of a standard orifice reduces the pressure loss [12].

Shape design optimization is an approach to obtain the geometric shape following the criteria of performance with both geometric and restriction. There are two basic approaches in the engineering optimization, Fig. 2. First, the surrogate based optimization where the surrogate model (such as artificial neural network) used to estimate the objective function during optimization. The surrogate-based method is intended to facilitate the optimization of a wide variety design problems. However this method is time consuming if the numbers of variables 
is large. A design optimization using the Kriging surrogate model is developed in multiple wing sails to maximize the thrust coefficient [13]. Surrogate-based analysis and optimization is conducted by Changli et al. [14] on an axisymmetric body.

On the other hand, the second optimization approach is the adjoint method. Adjoint method has been used in many engineering applications, where no surrogate model is needed. The adjoint method is a gradient-based approach which calculate the sensitivities regardless the number of design variables. What results a considerable saving in time and computational cost. Reducing the pressure drop of a novel gas cyclone was investigated using the discrete adjoint method [15]. In hydraulic parts, optimization of the design for a pressure compensator to reduce pressure drop was investigated [16]. Tonomura et al. [17] conducted a shape optimization of microchannel using the adjoint method. The shape of train's nose was optimized by using adjoint method to reduce the drag on train [18]. It is shown above that little researches take in consideration using adjoint method in internal flow. However, the present work introduces new shape of orifice plate by using the adjoint solver. The new design could reduce the pressure loss of standard orifice.

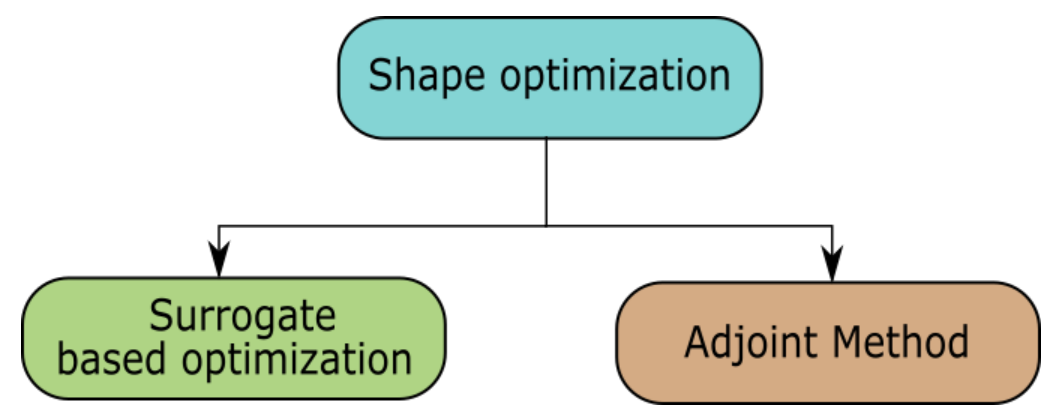

Fig. 2. Optimization strategy

\section{Aim of the present work}

The present research aims at minimizing the pressure loss associated with the standard orifice. Shape optimization based on adjoint method is applied for three studies as shown in Fig. 3. First study, the adjoint method is applied on a single orifice plate in order to control the flow convergence as represented in Fig. 3(a). However, using a downstream ring of a standard orifice plate shows a reduction in the pressure loss, Fig. 4 . The ring has a diameter ratio, $\beta_{r}$ of 0.5 oriented at a space, $l_{r} / D$ equals 0.38 . Hence, applying the adjoint method on an orifice plate with downstream ring was investigated. The second study objects to explore the optimal shape of a ring by controlling the pressure recovery after contraction process, Fig. 3(b). The third study aims at obtaining the optimal shape of orifice where the ring is in its original shape, Fig. 3(c).

To validate the present numerical simulation, the orifice geometry used in the present work is that experimentally studied by Nail (cited in Eiamsa [5]). The pipe has a diameter $D$ of $50.8 \mathrm{~mm}$, the orifice is $3.2 \mathrm{~mm}$ thick with a diameter ratio, $\beta$ of 0.5 and a Reynolds number $R_{e}=1.84 \times 10^{4}$. 


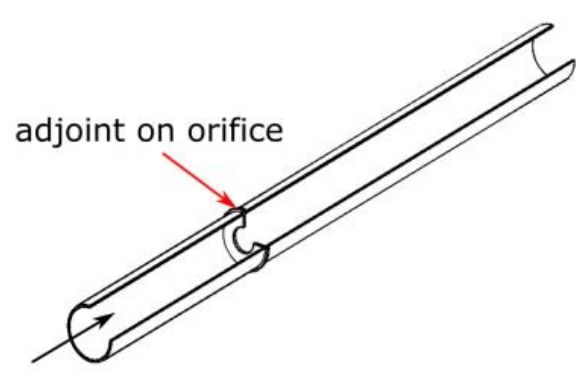

(a)

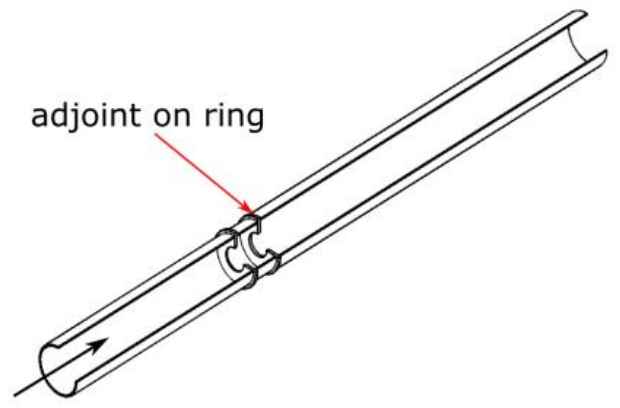

(b)

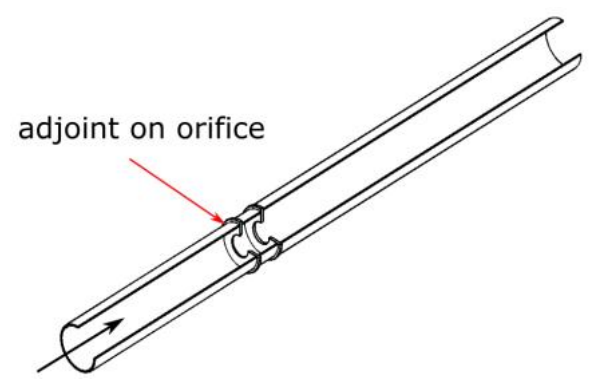

(c)

Fig. 3. Optimization test cases

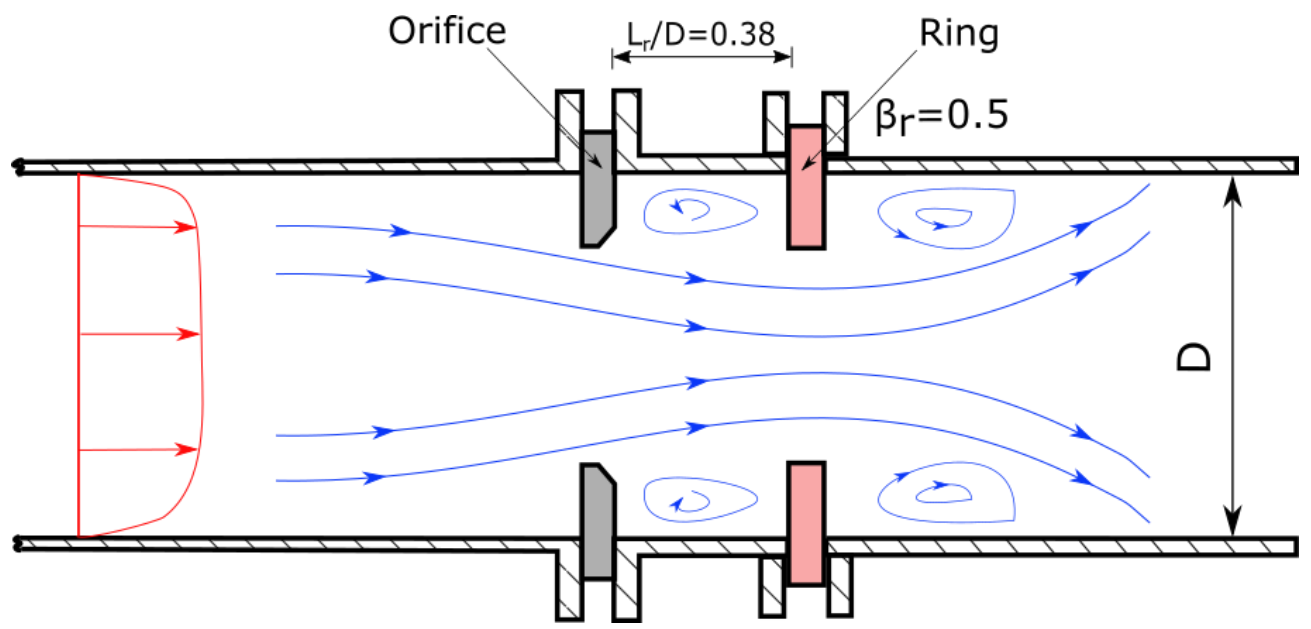

Fig. 4. Orifice plate with downstream ring

\section{CFD Modeling}

The present considered 3D steady simulation using Reynolds Averaged Navier-Stokes equations (RANS). Standard $k-\varepsilon$ turbulence model (SKE) was used which is the only supported turbulence model in the adjoint solver used. The discretization process is performed by the finite volume technique using the CFD code. The applied discretization schemes are second order scheme for pressure, SIMPLEC algorithm for pressure-velocity coupling, second order upwind scheme for momentum and first order upwind scheme for kinetic energy $k$ and turbulence dissipation rate $\varepsilon$. Incompressible fluid is set as dry air at $25^{\circ} \mathrm{C}$ and 1 atm with density $\rho=1.225 \mathrm{~kg} / \mathrm{m}^{3}$ and dynamic viscosity $\mu=1.831 \times 10^{-5} \mathrm{~Pa}$ s. Convergence criteria is completed when the residuals of mass, momentum and turbulence are less than $10^{-10}$. Furthermore, the inlet static pressure was monitored during the simulation process to validate the convergence. Moreover, the mass flux through the domain is inspected to validate the convergence. 


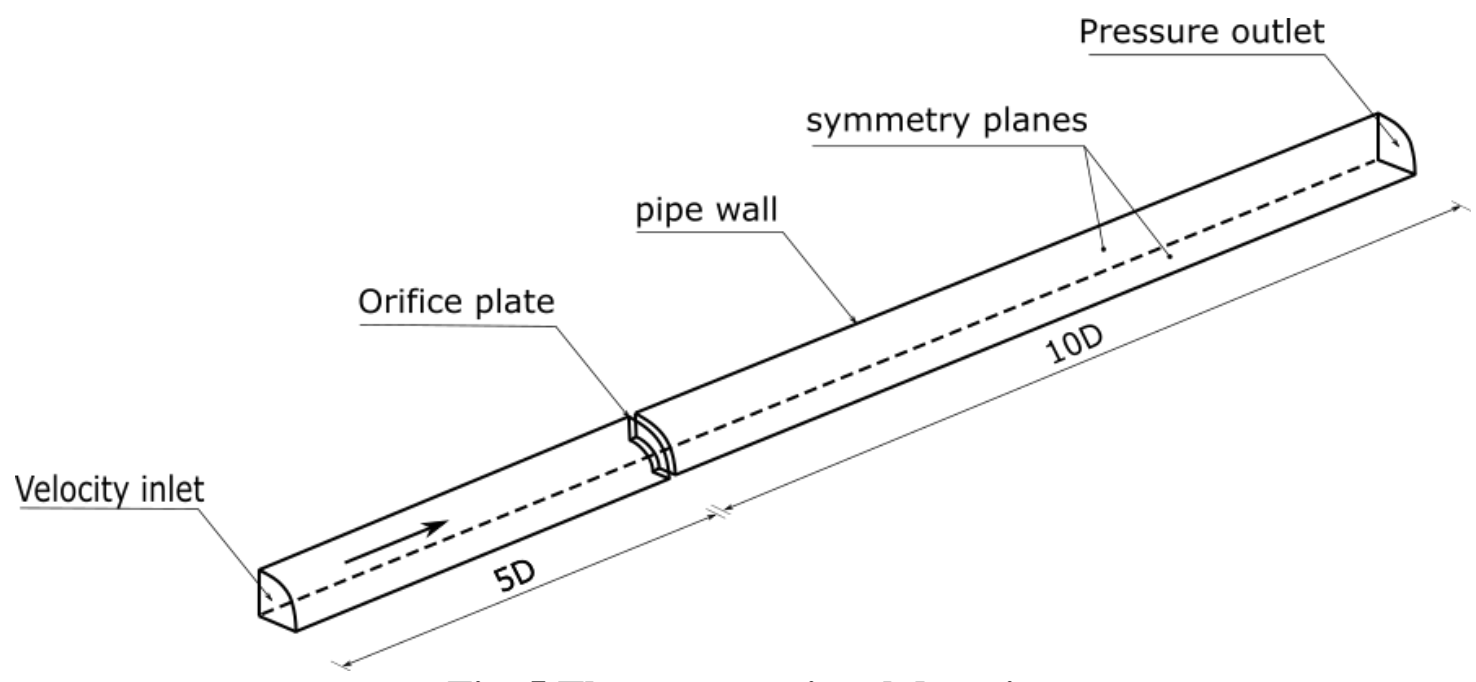

Fig. 5 The computational domain

The computational domain is extended 5 times and 10 times the pipe diameter upstream and downstream the orifice plate respectively, Fig. 5. For turbulent flow, it is acceptable to assume a uniform flow at the inlet section. However, it is acceptable to assume a uniform flow at the inlet section for turbulent flow. Uniform inlet velocity and pressure outlet boundary condition are employed for the computational domain inlet and outlet, respectively. The fluid flow is considered to be symmetrical about its axis. To reduce the computational time, only quarter of the complete domain was studied. Symmetry boundary conditions are utilized at horizontal and vertical planes. Wall boundary condition is applied at the pipe surface.

Structured hexahedral grids were utilized in the discretization of the flow domain. The mesh was constructed where cells near the wall are intense, Fig. 6. Enhanced wall treatment (EWT) was utilized in turbulence model because it uses a blending function near the wall. The first layer $y^{+}$was inspected at the wall all over the domain, Fig. 7. It is shown that $75 \%$ of the cells at the wall lie at viscous sublayer $\left(y^{+}<5\right)$ of turbulent boundary layer. Thus, using EWT can be considered as a good approach near the wall.

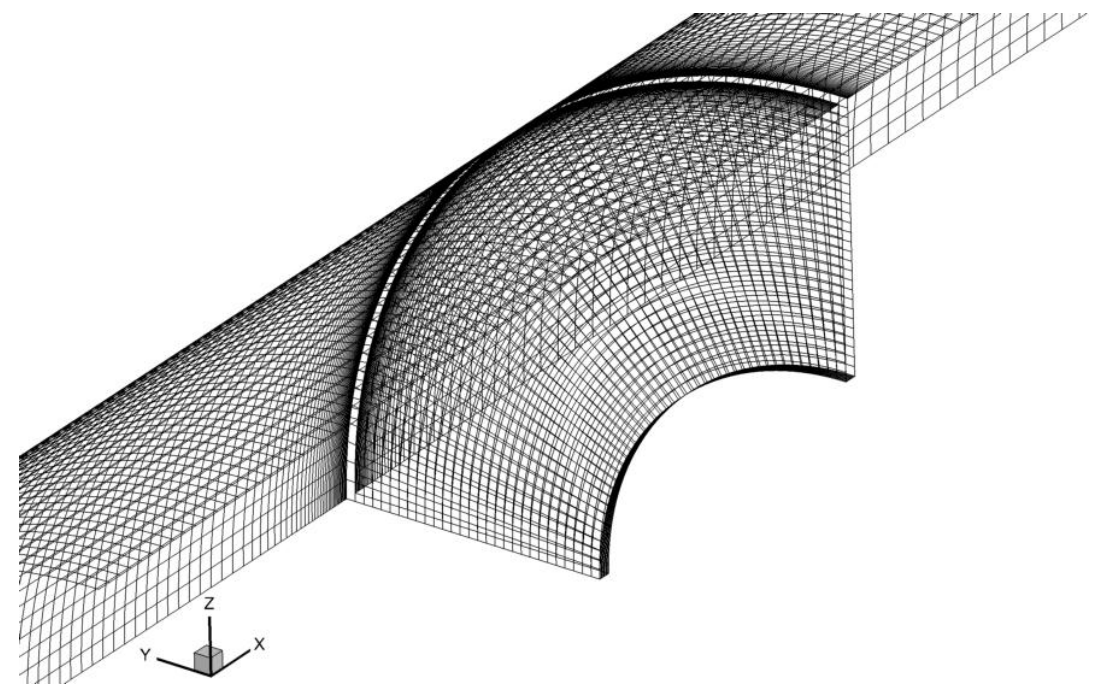

Fig. 6. A zoom around the orifice mesh 


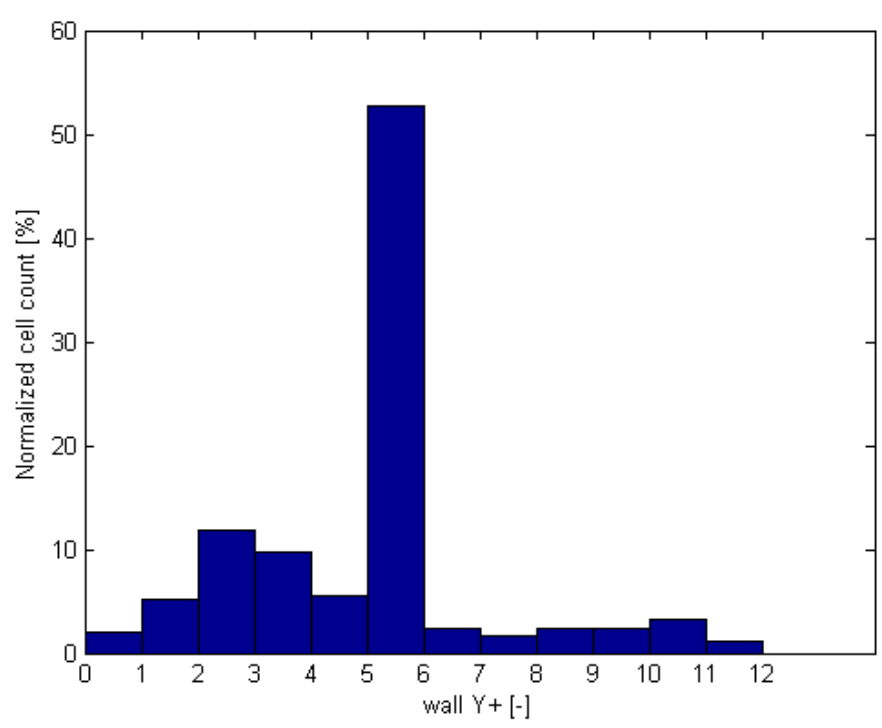

Fig. 7. $y^{+}$histogram at pipe wall

Mesh independent study was implemented by varying the number of mesh elements. Details of the grid independence study are presented in Table 1. Mesh independent study for the orifice plate discharge coefficient $C_{d}$ as a key parameter. The pressure taping ( $D$ and $D / 2$ ) was utilized upstream and downstream orifice, respectively. It is clear that the differences in the results are small between the three meshes. In further work, the medium mesh $(581,252)$ will be used to save computational time.

Table 1. Mesh independent study

\begin{tabular}{c|c|c}
\hline \hline Mesh size & $C_{d}$ & Difference $(\%)$ \\
\hline 445,345 & 0.6545 & $1.05 \%$ \\
581,252 & 0.6615 & $0.26 \%$ \\
693,025 & 0.66326 & -- \\
\hline
\end{tabular}

Furthermore, variation of the normalized pressure is examined along the pipe axis as another test Fig. 8 illustrates that the obtained results on the medium mesh is grid independent study. Good quantitative and qualitative results are achieved for numerical and experimental results.

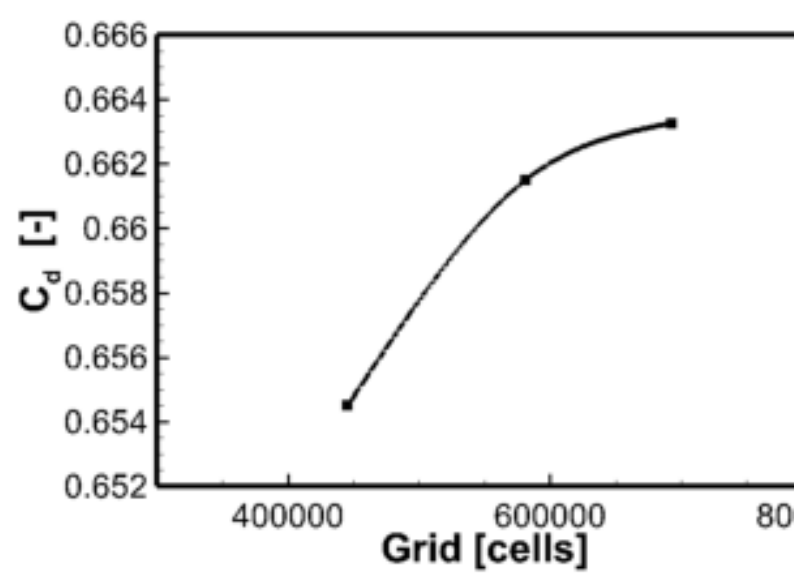

(a)

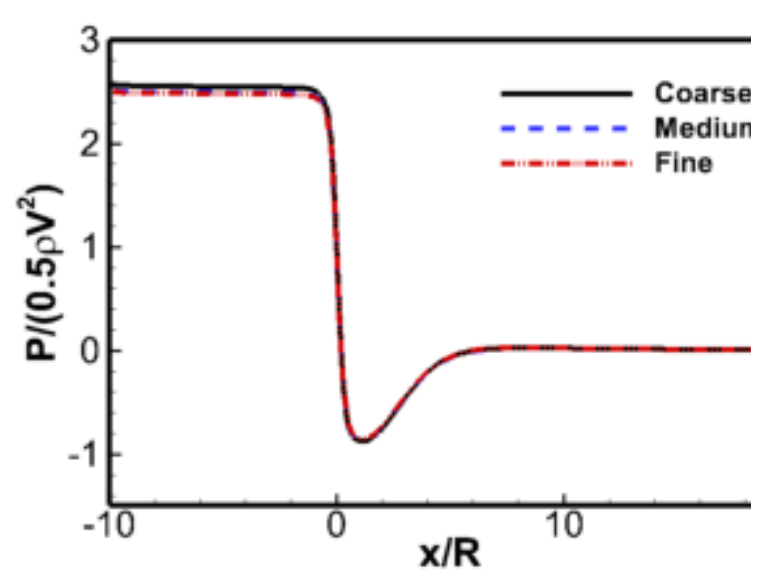

(b)

Fig. 8. Mesh independence study

(a) The discharge coefficient. (b) The Normalized axial pressure 
Validation of the numerical simulation with experimental results [5] was achieved as indicated in Fig. 9. The upstream surface of the orifice plate is referenced at $(x / R=0)$. The beak point of maximum velocity is beyond orifice at vena contracta section $(x / R=1)$. Fig. 9 shows the normalized axial velocity and the normalized pressure of numerical results compared with the experimental data. The deviations of the normalized axial velocity and the normalized pressure are less than 5\% from the experimental results.

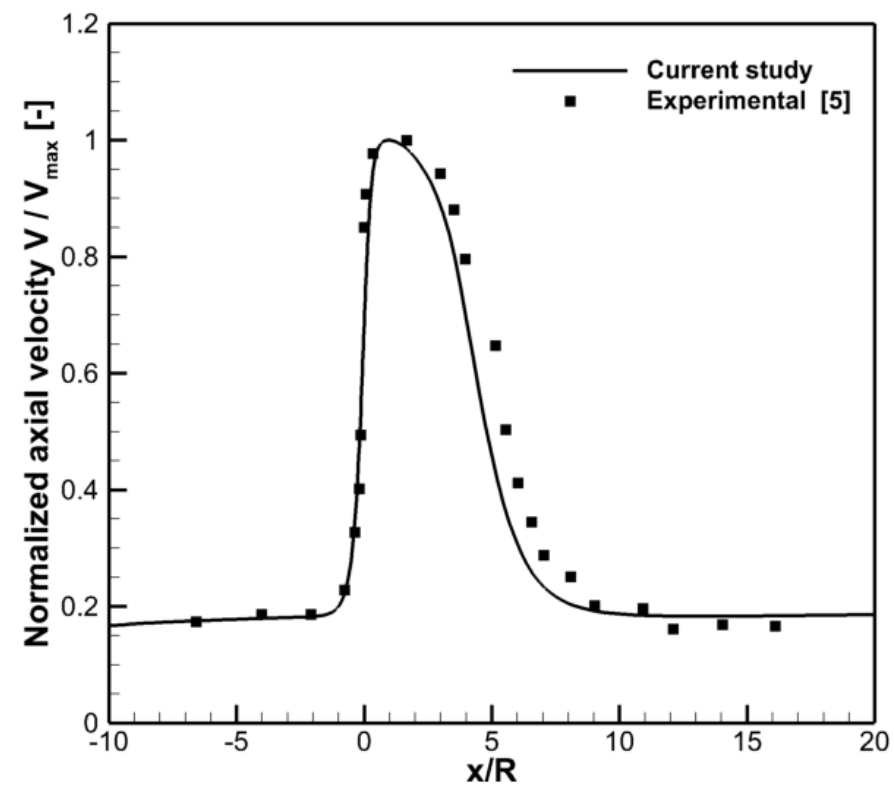

(a)

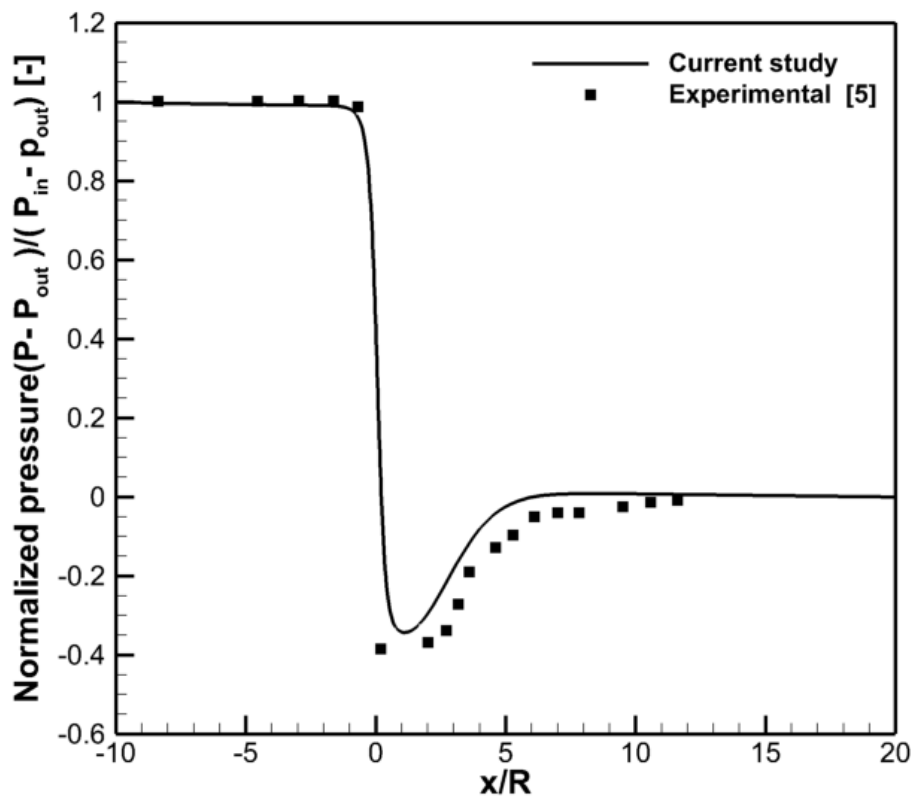

(b)

Fig. 9. Validation with experimental data (a) Normalized axial velocity. (b) Normalized static pressure. 


\section{Optimization Using the Adjoint Method}

Shape optimization process becomes a challenge when large numbers of parameters to be varied using surrogate based optimization. Adjoint method is a gradient-based optimization. The calculation of the sensitivities is not dependent on the numbers of design variables. Consequently, the adjoint method is computationally efficient. There are two types of the adjoint solvers, the continuous and the discrete methods. A continuous adjoint solver relies heavily on the mathematical properties of the partial-differential equations that define the physics of the problem. Discrete adjoint solver is based not on the form of the partial differential equations governing the flow, but the particular discretized form of the equations used in the flow solver itself. This has been observed to yield sensitivity data that provides valuable engineering guidance for the classes of problem. The discrete adjoint approach has been adopted for the ANSYS Fluent adjoint solver which has been used in this study.

Reduction of the pressure loss $(\mathcal{J})$ is the objective of this study. The design variables $(x)$ are used to control the shape. For a cell-centered finite-volume scheme, the flow variables $(q)$ are defined at the cell centroids by a vector of real values.

At convergence the flow variables satisfy the Navier-Stokes equations

$$
\mathcal{R}(q, x)=0
$$

Let the objective function,

$$
\mathcal{J}=\mathcal{J}(q, x)
$$

The adjoint method used to compute the sensitivity of the objective function (pressure loss) with respect to the control variables. Minimization of $\mathcal{J}$ requires computation of the gradient.

$$
\delta \mathcal{J}=\frac{\partial \mathcal{J}}{\partial q} \delta q+\frac{\partial \mathcal{J}}{\partial \mathrm{x}} \delta x
$$

If changes occur in the control variables, the flow variables change, which indirectly changes the objective function value. The change in flow variables $\delta q$ must satisfy

$$
\frac{\partial \mathcal{R}}{\partial q} \delta q=-\frac{\partial \mathcal{R}}{\partial \mathrm{x}} \delta x
$$

Equation (4) is used to determine the change in flow variables $(\delta q)$, whenever change in design variables $(\delta x)$. However, solving this equation is a time consuming for a handful of changes in design variable. Consequently, this tricky was resolved by eliminating the variations of the flow solution from equation (3). The adjoint method provides a mechanism for eliminating the changes that happen in the flow variables $(\delta q)$, when the design variables $(\delta x)$ changes. This is accomplished by introducing the adjoint variable $\psi$ to equation (1). This results in a relationship

$$
\left[\psi \frac{\partial \mathcal{R}}{\partial q}\right] \delta q=-\psi \frac{\partial \mathcal{R}}{\partial \mathrm{x}} \delta x
$$

he term in square brackets is matched with the left term in equation (3)

$$
\left[\psi \frac{\partial \mathcal{R}}{\partial q}\right]=\frac{\partial \mathcal{J}}{\partial q}
$$


This is the adjoint equation. The senistivty of the pressure drop was calculated with respect to the normal displacement of each node on the surface mesh. The procedures considered here to optimize orifice or ring using the adjoint method is represented in Fig. 10. After the flow solver is converged, the adjoint solver is used to calculate the sensitivity. Determination sensitivity is used to highlight the regions that have strong effect on the objective function. Thus, small change in these regions has high effect on the pressure loss. After that the adjusted region (orifice or ring) is selected to be modified by mesh morpher. The mesh morpher provides a smooth movement of the surface nodes with respect to the control points. The movements of the points are described by the direction that reduces the pressure drop. The recent shape is inspected by the flow solver. Finally, the convergence of optimal shape is determined by calculating the pressure drop after each cycle.

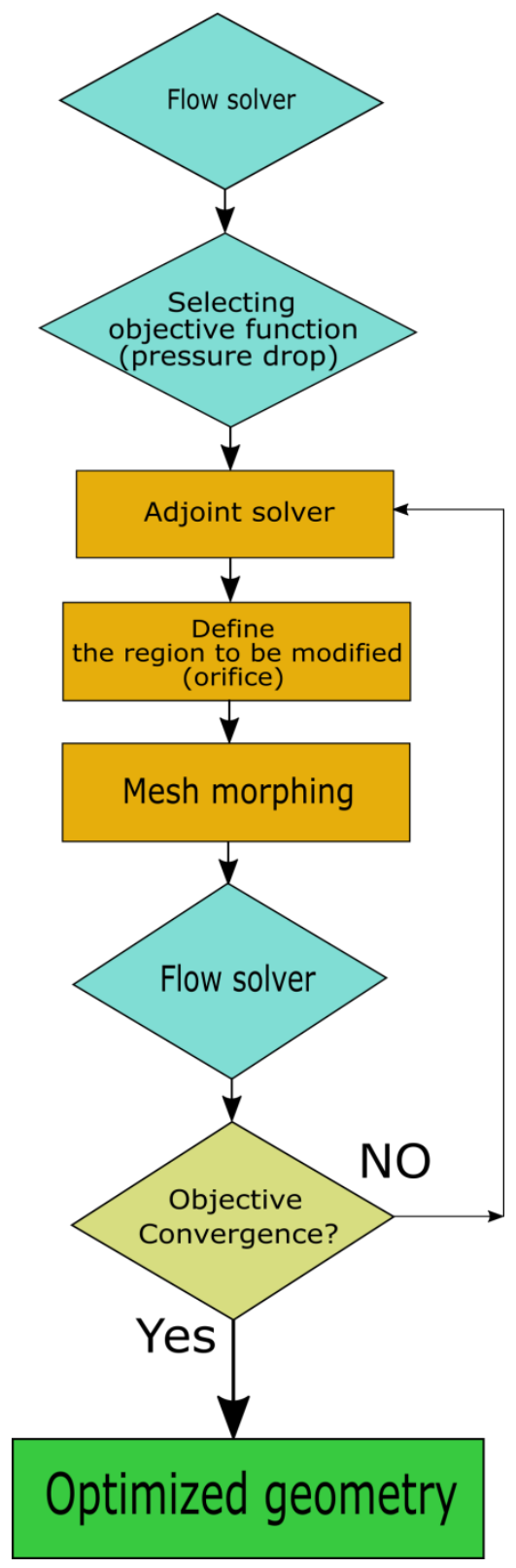

Fig. 10. Optimization process 


\section{Results and Discussion}

As shown in Fig. 11, high sensitivity around the orifice indicates where the modification in the shape should be focused. However, investigations were studied in orifice and ring respectively. Checking mesh quality was examined after deformation process. Repeating the optimization process until the changes in the shape has negligible change on the objective function.

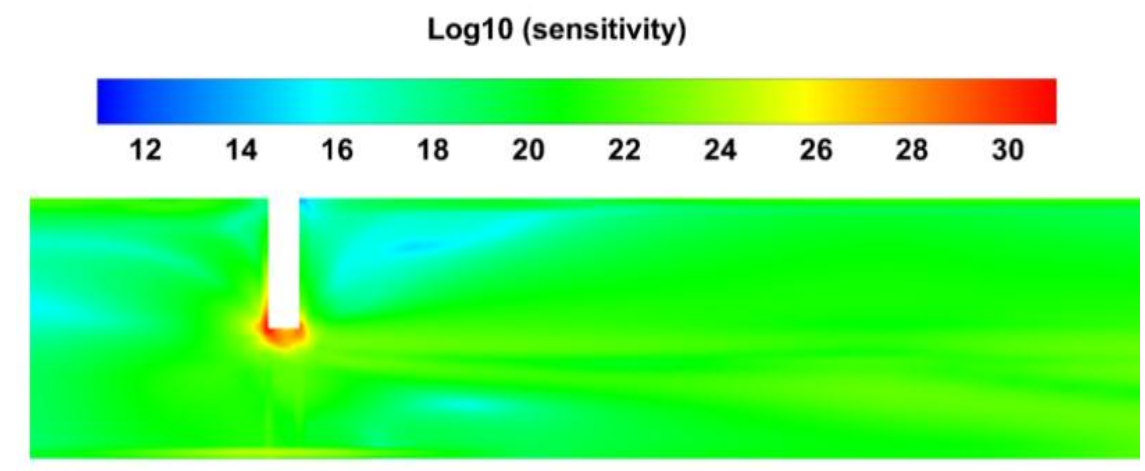

(a) $\log 10$ sensitivity for the orifice plate (case 1)

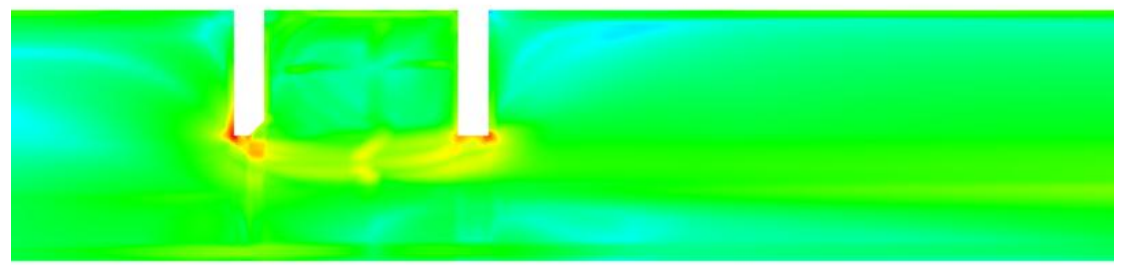

(b) $\log 10$ sensitivity for the orifice and the ring (case 2 and case 3 )

Fig. 11. Sensitivity data

(a) For orifice plate. (b) For orifice with ring

Discharge coefficient $\left(C_{d}\right)$ is widely used to estimate the performance of orifice flow meter. $\left(C_{d}\right)$ depends on the velocity coefficient $\left(C_{v}\right)$ and contraction coefficient $\left(C_{c}\right)$ where

$$
\begin{aligned}
& C_{v}=\frac{V_{j}}{V_{i}} \\
& C_{c}=\frac{A_{j}}{A}
\end{aligned}
$$

where $V_{j}$ is the jet velocity, $V_{i}$ is the ideal jet velocity, $A_{j}$ is the area of jet and $A$ is the area of orifice. At high $R_{e}$, the orifice $C_{d}$ is much more affected by variations in $C_{c}$ than it is by it is by variations in $C_{v}$. Keep in mind that $C_{c}$ is influenced by the shape of orifice plate. Determination of orifice $C_{d}$ was calculated according to the prescribed installation guide of ISO 5167-2:2003 at $R_{e}=1.84 \times 10^{4}$. It is the ratio of the actual mass flow rate $m_{a c}$ to the ideal mass flow $m_{i}$ rate through the orifice meter,

$$
C_{d}=\frac{m_{a c}}{m_{i}}
$$

The fluid flow through the orifice meter was considered incompressible. Thus, the formula used to calculate $m_{i}$ is 


$$
m_{i}=\frac{1}{\sqrt{1-\beta^{4}}} \frac{\pi}{4} d^{2} \sqrt{2 \rho(\Delta p)}
$$

where $d$ is the diameter of the orifice plate and $\Delta p$ is the pressure drop across the orifice meter. The $D$ and $D / 2$ pressure tapping was employed in the calculation. Table 2 compares the optimal orifice shape and the standard orifice. The application of the adjoint method improved the discharge coefficient $\left(C_{d}\right)$ for the three tested cases. However, it powerful effect contributes in increasing $\left(C_{d}\right)$ in case 1 by $39.9 \%$ at $R_{e}=1.84 \times 10^{4}$.

Table 2. Discharge coefficient calculation $R_{e}=1.84 \times 10^{4}$

\begin{tabular}{c|c|c|c}
\hline \hline Case no. & Baseline $\left(C_{d}\right)$ & Optimal $\left(C_{d}\right)$ & Difference (\%) \\
\hline Case 1 & 0.65455 & 0.91584 & $39.9 \%$ \\
Case 2 & 0.75788 & 0.77408 & $2.1 \%$ \\
Case 3 & 0.75788 & 0.89539 & $18.1 \%$ \\
\hline \hline
\end{tabular}

Also, the pressure loss is estimated with respect to the installation guide of ISO 5167-2:2003. The pressure loss $(\Delta \omega)$ is the static pressure difference between the measured pressure at $D$ upstream of the orifice and the pressure at $6 D$ downstream of the orifice, Fig. 12.

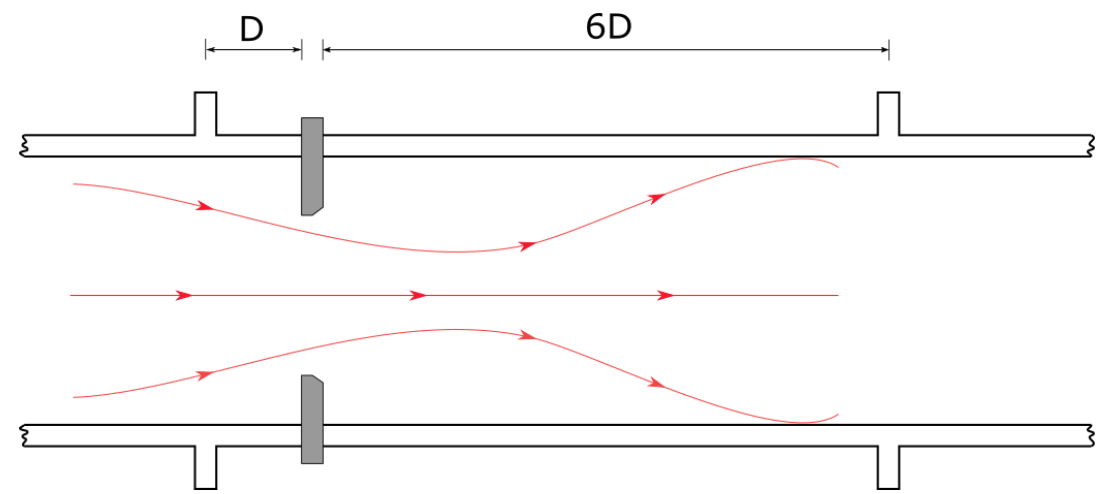

Fig. 12. Pressure loss taps

The pressure loss coefficient $K$ is expressed as (ISO 5167-2:2003)

$$
K=\frac{\Delta \omega}{\frac{1}{2} \rho V^{2}}
$$

where $\Delta \omega$ is the pressure loss and $V$ is the mean axial velocity of fluid in the pipe. Measuring pressure loss coefficient for three cases is described in Table 3. Employing the adjoint method in shape optimization reflects satisfied pressure loss up to 11.37 in case 1 . Reduction in pressure loss (case 1 and case 3 ) is achieved owing to controlling jet contraction. Smooth jet contraction prevents formation small area of vena contracta. 
Table 3. Pressure loss coefficient $K$ for three cases

\begin{tabular}{c|c|c|c}
\hline \hline Case no. & Baseline $(K)$ & Optimal $(K)$ & Difference $(\%)$ \\
\hline Case 1 & 25.22 & 11.37 & $-54.91 \%$ \\
Case 2 & 18.74 & 18.22 & $-2.77 \%$ \\
Case 3 & 18.74 & 12.35 & $-34.09 \%$ \\
\hline \hline
\end{tabular}

Fig. 13 shows the final shapes that were produced by the adjoint method. Converged shape (case 1 and case 3 ) provides more control on formation of vena contracta area. The larger cross section area is of vena contraction, the higher value of contraction coefficient $\left(C_{c}\right)$. Subsequently, discharge coefficient $\left(C_{d}\right)$ increases in case of using the converged shape (case 1 and case 3 ). On the other hand, different shape was produced when investigation was applied on downstream ring (case 2). The optimal shape of ring acts as diffuser that helps in recovery pressure after contraction process, as shown in Fig. 13.

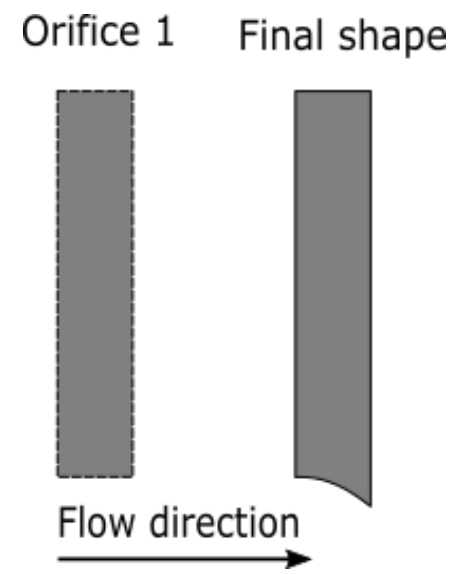

(a) Case 1

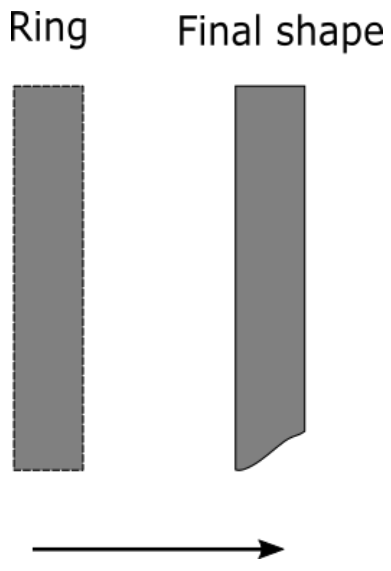

(b) Case 2

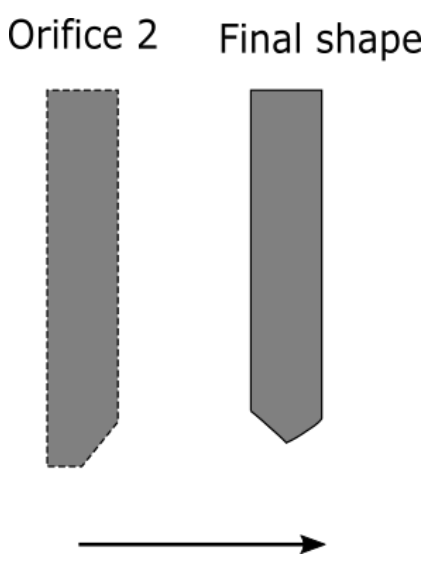

(c) Case 3

Fig. 13. Optimal shape for the orifice and the ring

Fig. 14 presents the vorticity contours for case 1(orifice 1). Vorticity is a measure of the rate of rotation in a fluid which plays an important effect on the pressure loss. High vorticity exists near the tip of the orifice, due to the flow separation at the right angle of the orifice plate. As shown in Fig. 14, the shape is changed in the flow direction to minimize the separation zone. As a result, the pressure losses decreased by reducing the vorticity magnitude. 


\section{Vorticity}

50001000015000200002500030000350004000045000

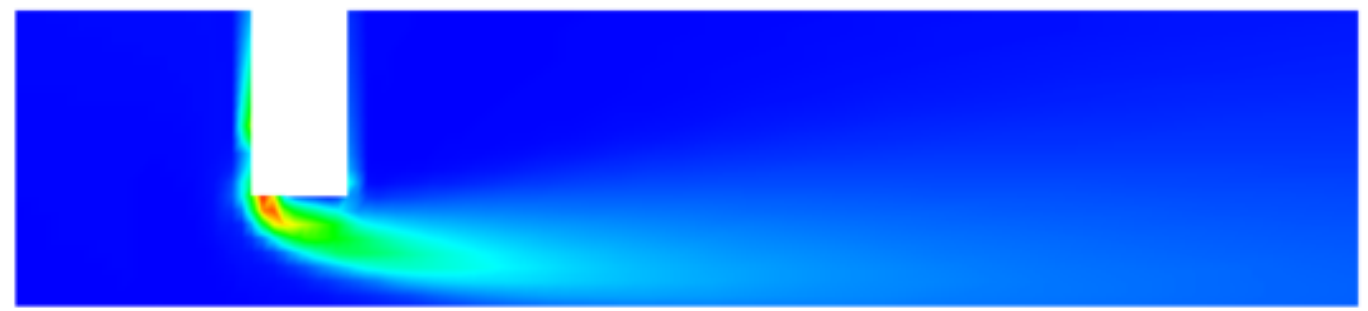

(a) Baseline shape (orifice 1)

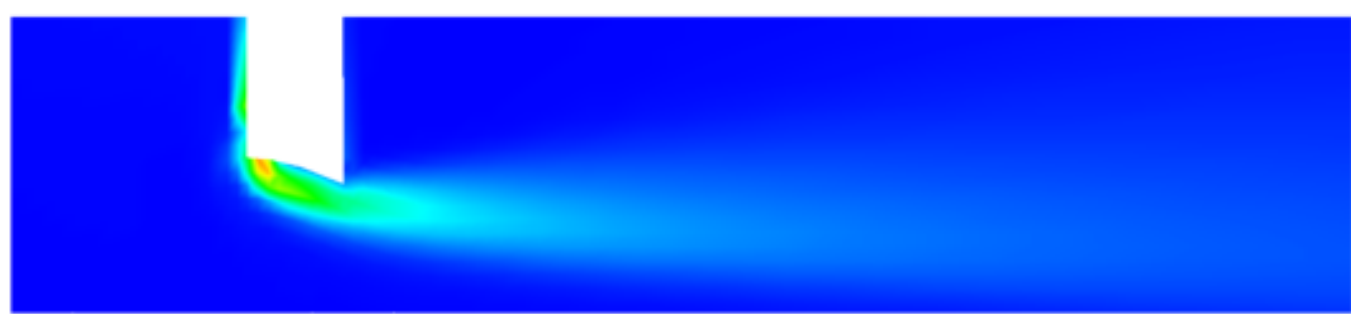

(b) Optimal shape (orifice 1)

Fig. 14. Vorticity contour

In case 2 and case 3, a downstream ring with the orifice meter was studied. Shape optimization of ring (case 2) and orifice (case 3) was demonstrated and presented in Fig. 15. The optimal shape of orifice 2 (case 3 ) has a more significant effect than the optimal ring (case 2) in reduction the vorticity magnitude. Moreover, this reflects on enhancement of the discharge coefficient $\left(C_{d}\right)$ by the reducing pressure drop.

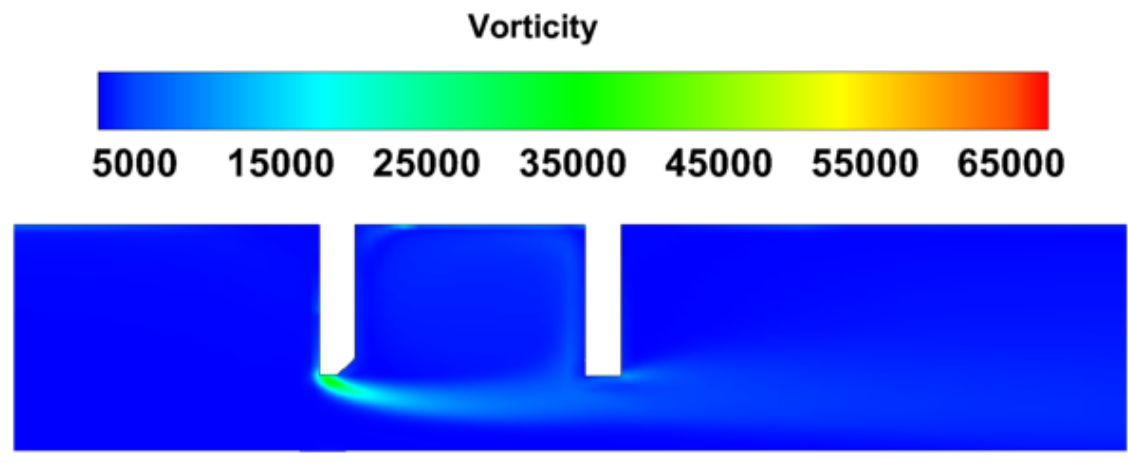

(a) Case 2 and case 3 before optimization.

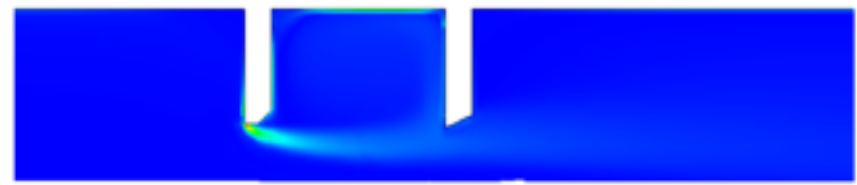

(b) optimal ring

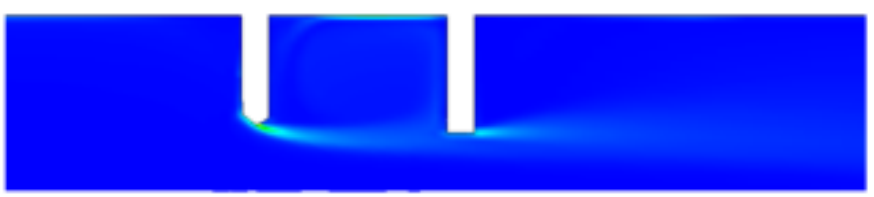

(b) optimal orifice 2

Fig. 15. Vorticity contour case 2 and 3 
The velocity contour was explored for case 1 at $R_{e}=1.84 \times 10^{4}$ as shown in Fig. 16. It is obvious that the optimal orifice (case 1) produce jet velocity lower than the standard orifice. Smooth jet contraction contributes in increasing the contraction coefficient $\left(C_{c}\right)$ because of higher area of vena contracta, consequently the discharge coefficient $\left(C_{d}\right)$ increases.

Pressure loss occurs in orifice meter can be classified based on the contraction effect of vena contracta and uncontrolled expansion. Showing the effect of the shape optimization (orifice 2 and a ring) on decreasing the pressure loss during measuring process is clarified on Fig. 17. Higher velocity regions illustrate the way of configuration the vena contracta. Optimal shape of orifice 2 has strong influence on elimination the construction of vena contracta that lead to reducing the pressure losses. Otherwise, optimal shape of ring has slight impact on the pressure loss as, the fluid flow exhibits higher velocity before the ring.

The velocity profiles of axial velocity at $(x / R=1)$ is studied to illustrate the effect of the optimal design of orifice plate as shown in, Fig. 18. The negative values of axial velocities (case 1) represent the effect of the recirculation zone behind the orifice plate. It is very clear that the converged shape of the orifice (case 1 and case 3 ) reduces the jet velocity. Moreover, the contraction coefficient $\left(C_{c}\right)$ is increased due to vena contracta effect is reduced.

$\mathrm{V}[\mathrm{m} / \mathrm{s}]$

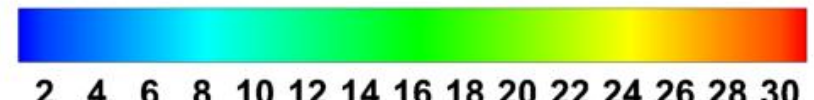

$\mathbf{x} / \mathbf{R}$
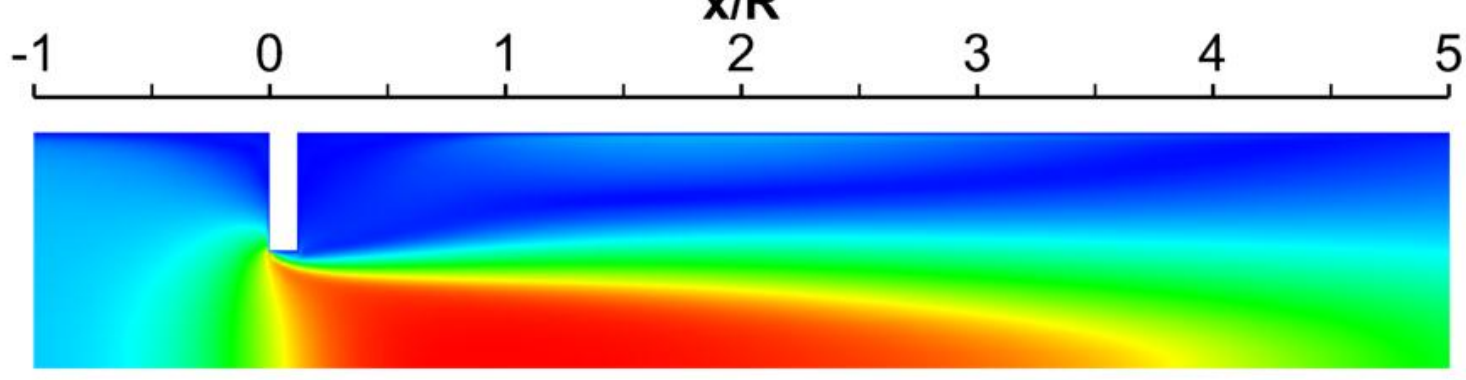

(a) Standard orifice plate

$\mathbf{x} / \mathbf{R}$

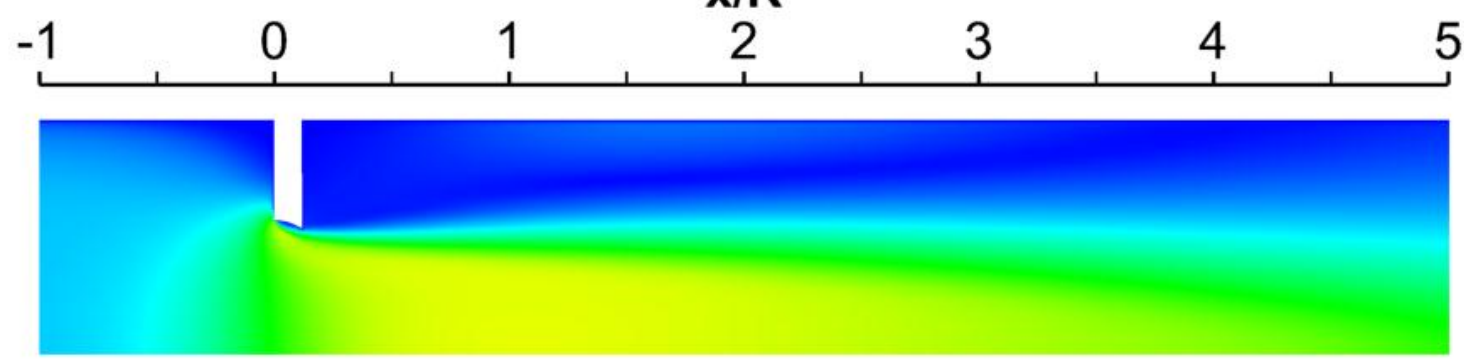

(b) optimal orifice plate

Fig. 16. Velocity contour of case 1 at $R_{e}=1.84 \times 10^{4}$. 


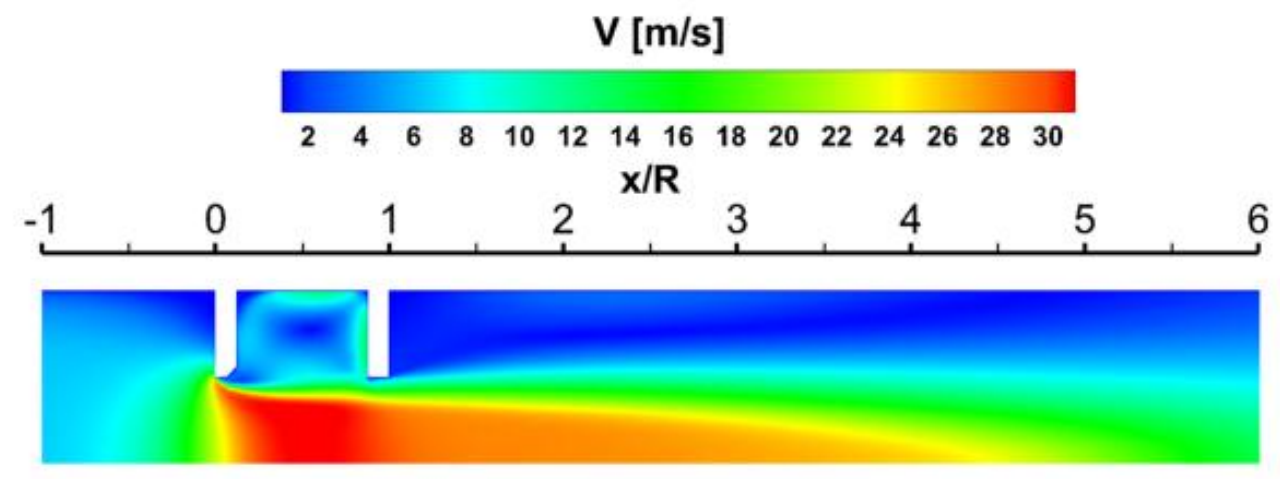

(a) Baseline shape of orifice 2 and a ring

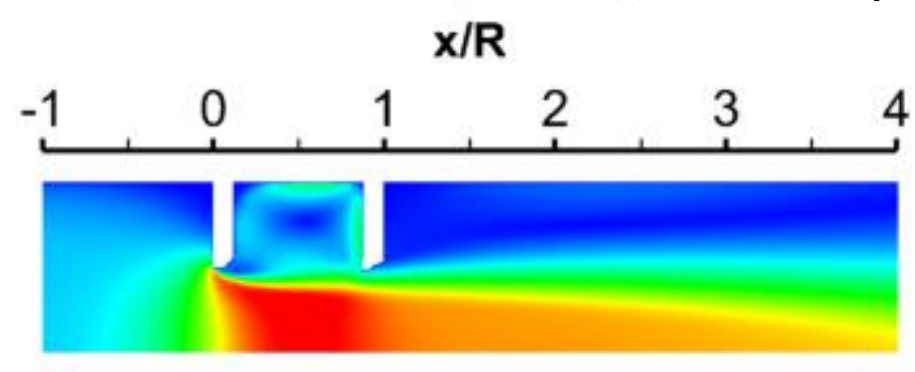

(b) optimal ring

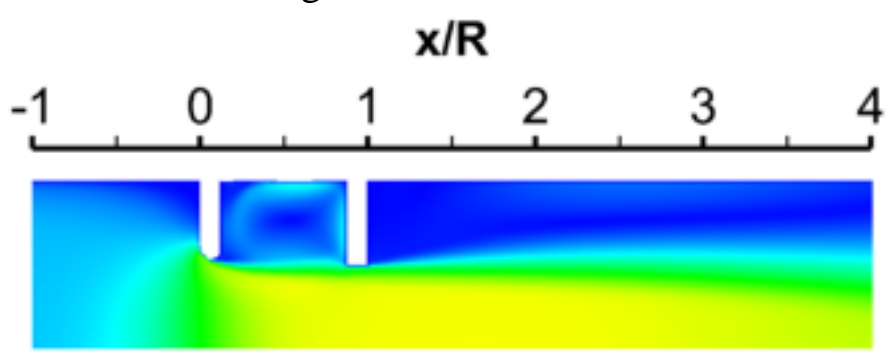

(c) optimal orifice 2

Fig. 17. Velocity contour for case 2 and case $3 R_{e}=1.84 \times 10^{4}$.

Variation of the static pressure with respect to the inlet dynamic pressure $\left(\frac{1}{2} \rho V_{\text {in }}{ }^{2}\right)$ is shown in Fig. 19. It is clear that the pressure loss is decreased throughout the optimal shapes (orifice 1 and orifice 2). It is concluded that the vena contracta has a strong effect on the energy loss. Thus, the smooth contraction with higher area of vena contracta reduces the pressure loss. On the other hand, the change in the ring shape (case 2) leads to a small variation of the pressure drop, Fig. 19.

\section{Conclusions}

Orifice meters are commonly used in industrial facilities and pipelines. The recent research aims at decreasing the permanent pressure loss and hence the energy consumption of standard orifice meters by using shape optimization. The adjoint method was employed in preforming shape optimization. Using ring $\beta_{r}$ of 0.5 oriented at a space $l_{r} / D$ equals 0.38 downstream the orifice plate aids in reducing pressure loss. However, the adjoint method was directed to get the optimal shape of orifice and ring. The results show that optimal shape of orifice $\beta$ of 0.5 has a strong effect in reducing the pressure loss and increasing the discharge coefficient $C_{d}$. Also, optimize the ring has slight change in performance. Increasing discharge coefficient $C_{d}$ by $39.9 \%$ and reducing pressure loss by $54.91 \%$ while the adjoint method on the orifice (case 1) without downstream ring at $R_{e}=1.84 \times 10^{4}$. 


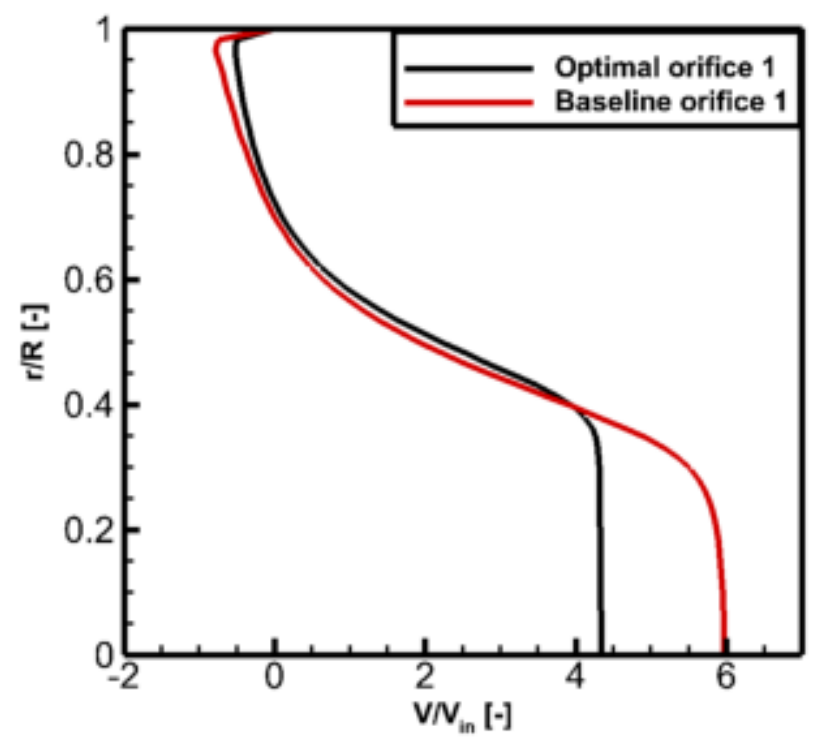

(a) case 1

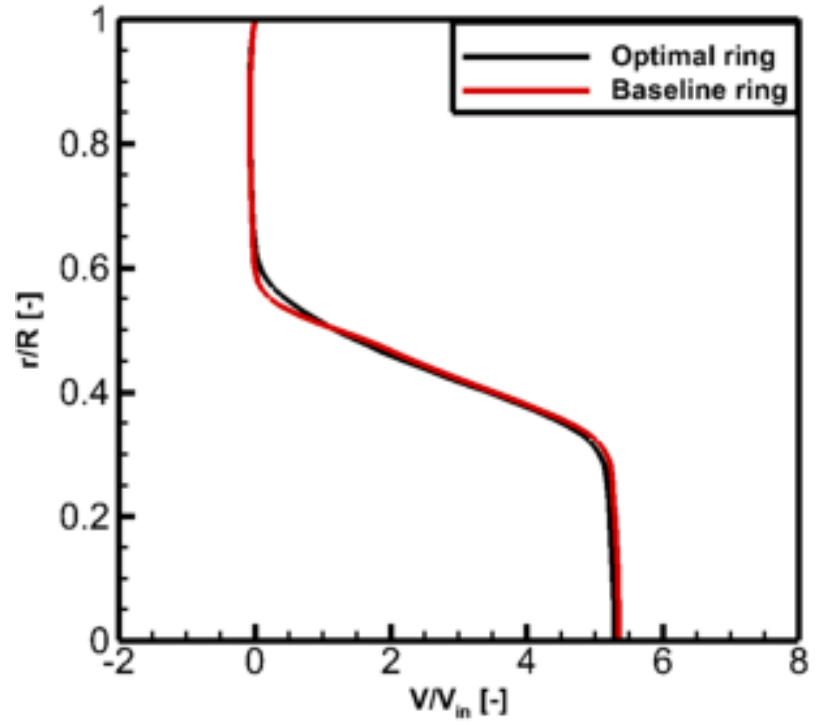

(b) case 2

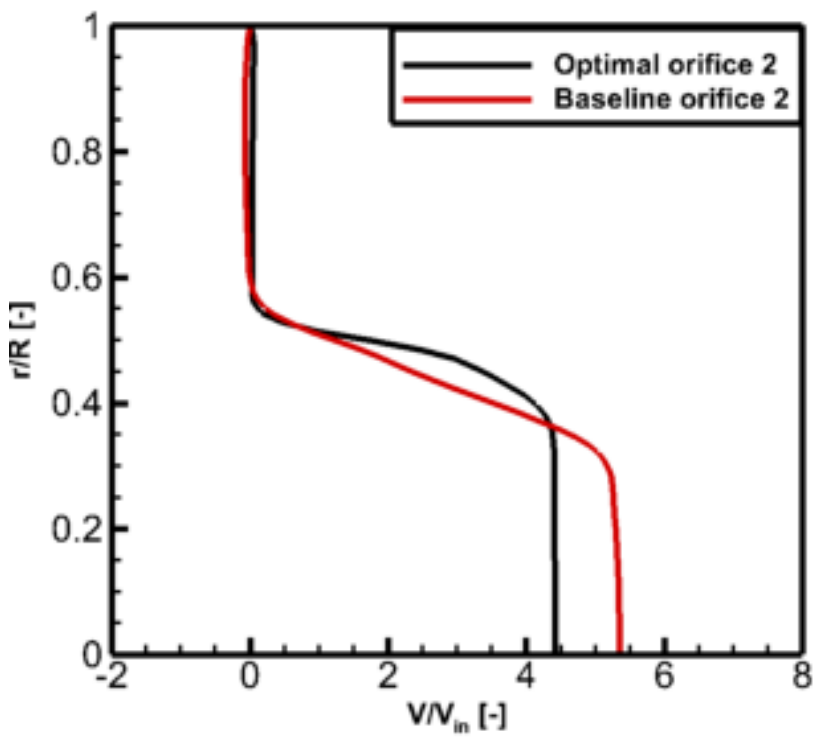

(c) case 3

Fig. 18. Velocity profiles at $x / R=1$ 


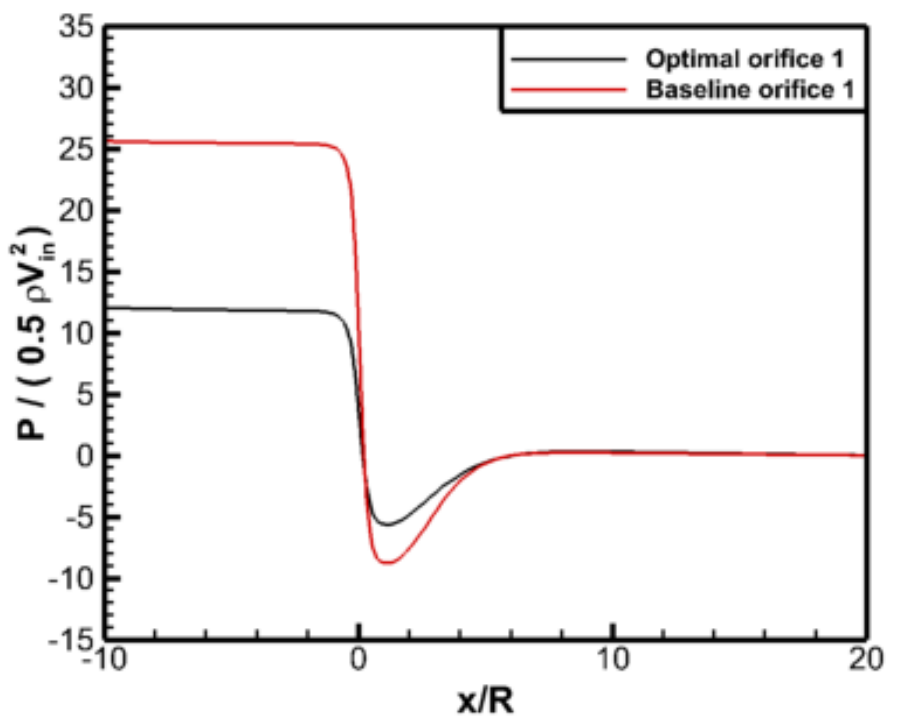

(a) case 1

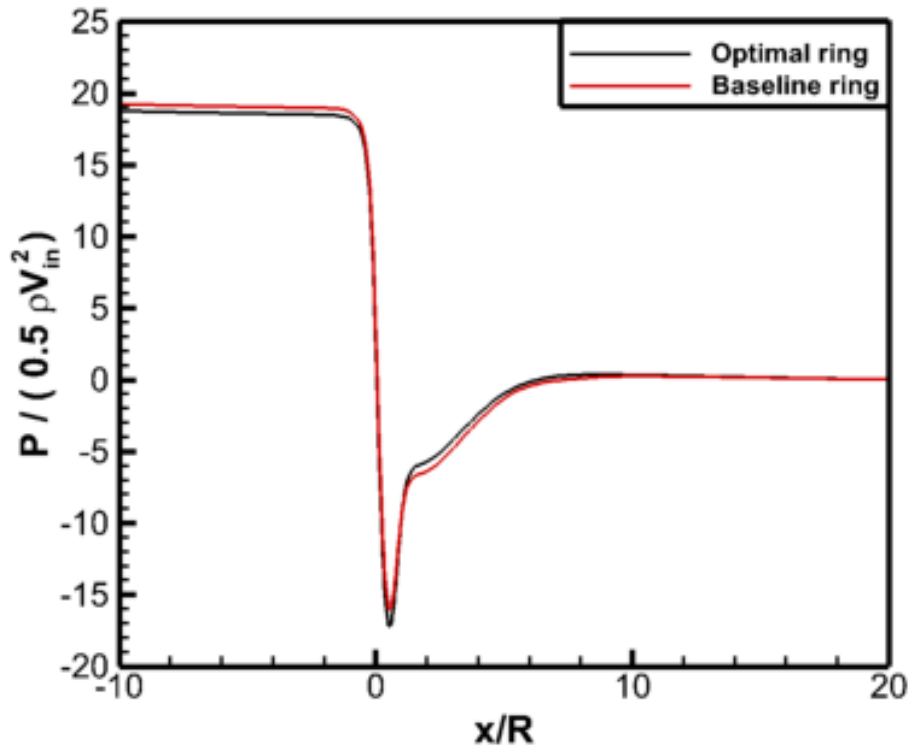

(b) case 2

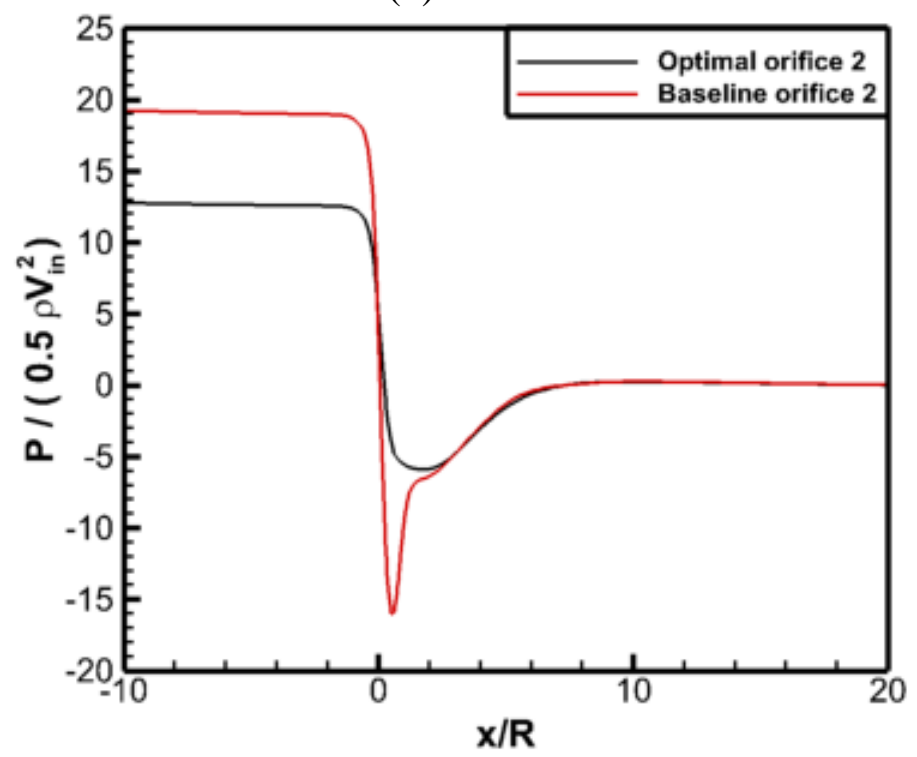

(c) case 3

Fig. 19. Normalized axial static pressure 


\section{References}

[1] Erdal, A. and Andersson, H. "Numerical aspects of flow computation through orifice", "Flow Measurement and Instrumentation", 1997, pp. 27-37.

[2] Tunay, T. "Investigation of the Effects of Different Numerical Methods on the Solution of the Orifice Flow" , "Cukurova University Journal of the Faculty of Engineering and Architecture ", 2012, pp. 39-51.

[3] Imada, F.H.J. and Saltara, F. "Numerical Determination of Discharge Coefficients of Orifice Plates and Nozzles" , " 22nd International Congress of Mechanical Engineering (COBEM 2013)", 2013, pp. 1755-1760.

[4] Mohan Kumar H M, Yogesh Kumar K J, D.V.S. "CFD Analysis of Flow through Dual Orifice Plate Assembly", 2015, pp. 136-144.

[5] Eiamsa-ard, S., Ridluan, A., Somravysin, P., Promvonge, P., and Chok, N. "Numerical investigation of turbulent flow through a circular orifice", " KMITL Sci.J. ", 2008, pp. 44-50.

[6] Siba, M.A., Mohd, W., Wan, F., Nuawi, M.Z., Rasani, R., and Mohamed, H. "Modeling and Applications of 3D Flow in Orifice Plate At Low Turbulent Reynolds Numbers", 2015, pp. 2-8.

[7] Shah, M.S., Joshi, J.B., Kalsi, A.S., Prasad, C.S.R., and Shukla, D.S. "Analysis of flow through an orifice meter: CFD simulation" , "Chemical Engineering Science ", 2012, pp. 300-309.

[8] Abou El-Azm Aly, A., Chong, A., Nicolleau, F., and Beck, S. "Experimental study of the pressure drop after fractal-shaped orifices in turbulent pipe flows", " Experimental Thermal and Fluid Science ", 2010, pp. 104-111.

[9] Huang, S., Ma, T., Wang, D., and Lin, Z. "Study on discharge coefficient of perforated orifices as a new kind of flowmeter" , "Experimental Thermal and Fluid Science ", 2013, pp. 74-83.

[10] Singh, V.K. and John Tharakan, T. "Numerical simulations for multi-hole orifice flow meter", " Flow Measurement and Instrumentation ", 2015, pp. 375-383.

[11] Shaaban, S. "On the performance of perforated plate with optimized hole geometry", "Flow Measurement and Instrumentation", 2015, pp. 44-50.

[12] Shaaban, S. "Optimization of orifice meter's energy consumption" , "Chemical Engineering Research and Design ", 2014, pp. 1005-1015.

[13] Lee, H., Jo, Y., Lee, D.-J., and Choi, S. "Surrogate model based design optimization of multiple wing sails considering flow interaction effect", " Ocean Engineering ", 2016, pp. 422-436.

[14] Changli, H., Wang, G., Chen, G., and Huang, B. "Surrogate model-based optimization for the headform design of an axisymmetric body" , "Ocean Engineering ", 2015, pp. 237-245.

[15] Elsayed, K. "Design of a novel gas cyclone vortex finder using the adjoint method", "Separation and Purification Technology", 2015, pp. 274-286.

[16] Petrovič, M. "Optimization of hydraulic parts using adjoint optimization" , "Perspectives in Science", 2016, pp. 337-340.

[17] Tonomura, O., Kano, M., and Hasebe, S. "Shape optimization of microchannels using CFD and adjoint method", "Computer Aided Chemical Engineering ", 2010, pp. 37-42.

[18] Munoz-Paniagua, J., Garcia, J., Crespo, A., and Laspougeas, F. "Aerodynamic optimization of the nose shape of a train using the adjoint method", "Journal of Applied Fluid Mechanics", 2015, pp. 601-612. 